\title{
Compensatory Sprouting and Impulse Rerouting after Unilateral Pyramidal Tract Lesion in Neonatal Rats
}

\author{
Werner J. Z'Graggen, Karim Fouad, Olivier Raineteau, Gerlinde A. S. Metz, Martin E. Schwab, and \\ Gwendolyn L. Kartje \\ Brain Research Institute, University of Zurich and Swiss Federal Institute of Technology Zurich, CH-8057 Zurich, \\ Switzerland
}

\begin{abstract}
After lesions of the developing mammalian CNS, structural plasticity and functional recovery are much more pronounced than in the mature CNS. We investigated the anatomical reorganization of the corticofugal projections rostral to a unilateral lesion of the corticospinal tract at the level of the medullary pyramid (pyramidotomy) and the contribution of this reorganization and other descending systems to functional recovery.

Two-day-old (P2) and adult rats underwent a unilateral pyramidotomy. Three months later the corticofugal projections to the red nucleus and the pons were analyzed; a relatively large number of corticorubral and corticopontine fibers from the lesioned side had crossed the midline and established an additional contralateral innervation of the red nucleus and the pons. Such anatomical changes were not seen after adult lesions.

Intracortical microstimulation of the primary motor cortex with EMG recordings of the elbow flexor muscles were used to inves-
\end{abstract}

tigate possible new functional connections from the motor cortex of the pyramidotomy side to the periphery. In rats lesioned as adults, stimulation of the motor cortex ipsilateral to the pyramidotomy never elicited EMG activity. In contrast, in P2 lesioned rats bilateral forelimb EMGs were found. EMG latencies were comparable for the ipsilateral and contralateral responses but were significantly longer than in unlesioned animals. Transient inactivation of both red nuclei with the GABA receptor agonist muscimol led to a complete loss of these bilateral movements. Movements and EMGs reappeared after wash-out of the drug. These results suggest an important role of the red nucleus in the reconnection of the cortex to the periphery after pyramidotomy.

Key words: red nucleus; basilar pontine nuclei; corticospinal tract; rubrospinal tract; stimulation; injury; motor system
In contrast to the situation in the mature CNS, reorganization and regeneration of fiber connections in response to a lesion can occur during development (for review, see Donoghue, 1995, 1997; Schwab and Bartholdi, 1996). Thus, several studies in different species showed that after unilateral neonatal cortical ablation corticofugal fibers from the opposite, spared hemicortex sprouted into the denervated contralateral red nucleus (Leong and Lund, 1973; Nah and Leong, 1976a,b; Naus et al., 1985a,b; Murakami et al., 1990, 1993), basilar pontine nuclei (Leong and Lund, 1973; Castro and Mihailoff, 1983; Kartje-Tillotson et al., 1986), and spinal cord (Castro, 1975; Kartje-Tillotson et al., 1985, 1987; Rouiller et al., 1991).

In another lesion paradigm, the unilateral transection of the developing corticospinal tract (CST) at the level of the medulla oblongata (pyramidotomy) in newborn hamsters, axons were found to project in aberrant pathways to target regions in the spinal cord (Kalil and Reh, 1982). Whether these axons are regenerated axons or redirected late-growing CST fibers remained controversial (Kalil and Reh, 1982; Tolbert and Der, 1987; Merline and Kalil, 1990). Functional testing after neonatal pyramidotomy in hamsters

Received Dec. 29, 1999; revised May 22, 2000; accepted June 14, 2000.

This study was supported by grants of the Swiss National Science Foundation, Berne, Switzerland (Grants 31-45549.95/2 and 4038-043918.95/2); the Biotechnology Program of the European Union, Bruxelles, Belgium; the Dr. Eric Slack-GyrFoundation, Zurich, Switzerland; the Spinal Cord Consortium of the Christopher Reeve Paralysis Foundation, Springfield, NJ; the International Research Institute for Paraplegia, Zurich, Switzerland; the Binelli-Ehrsam-Foundation, Zurich, Switzerland; the Velux-Foundation, Glarus, Switzerland, and the U.S. Department of Veteran Affairs. We thank our colleagues Drs. E. J. Neafsey, P. Streit, M.-C. Hepp-Reymond, and C. E. Bandtlow for their help, Drs. A. McKinney and J. Tönnes for valuable discussions, and M. Thallmair for helping with this manuscript. We also thank R. Schöb for photographic and E. Hochreutener for graphic support, Dr. R. Dürr, H. J. Kasper, and R. Kägi for their technical support, and S. Kaufmann for secretarial work.

Correspondence should be addressed to Werner J. Z'Graggen, Brain Research Institute, University of Zurich, Winterthurerstrasse 190, CH-8057 Zurich, Switzerland. E-mail: werner.zgraggen@ksa.ch.

Dr. Kartje's present address: Research Service, Hines Veterans Administration Hospital, Hines, IL 60141.

Copyright (C) 2000 Society for Neuroscience $0270-6474 / 00 / 206561-09 \$ 15.00 / 0$ showed high levels of improvement for forelimb manipulations of sunflower seeds but low recovery for locomotion (Reh and Kalil, 1982; Keifer and Kalil, 1991).

The anatomical reorganization of corticofugal pathways and connections rostral to a lesion of the CST in neonates have not been well studied. In addition, the contribution of lesion-induced neuroanatomical plasticity and the involvement of other descending fiber tract systems to functional compensations after neonatal lesion are unknown. The goal of this study was to investigate the lesion-induced neuroanatomical CST plasticity and its electrophysiological consequences 3 months after a pyramidal tract lesion applied to newborn rats.

Our results show a major reorganization of the projections of the affected cortex to two important brainstem targets: the red nucleus and the basilar pontine nuclei. Microstimulation of the affected cortex in these animals elicited forelimb EMGs that were abolished by injections of the GABA agonist muscimol into the red nuclei. Such responses were never observed in animals lesioned as adults.

\section{MATERIALS AND METHODS}

All animal experiments were performed under supervision of the cantonal veterinary department of Zurich, Switzerland. A total of 52 Lewis rats of either sex were included in this study. The animals were divided into the following groups: (1) unilateral pyramidotomy at postnatal day 2 (P2) (neonatal PTX; $n=29$ ), (2) unilateral pyramidotomy at adult age (3 months of age, adult PTX; $n=12$ ), and (3) unlesioned (3-4 months of age, $n=11)$.

In 24 animals the corticofugal anatomy was analyzed 3 months after lesion. At the same time point after lesion, the remaining 21 animals underwent electrophysiological testing.

\section{Pyramidotomy}

A unilateral pyramidotomy was performed to transect selectively the axons of the CST at the level of the caudal medulla oblongata (Fig. 1) (Kalil and Reh, 1982; Z'Graggen et al., 1998). At P2, rat pups were anesthetized by hypothermia and kept on ice during the entire surgical procedure. Using a ventral approach, the right pyramidal tract was exposed by retracting the paratracheal tissue and removing the bone overlying the medullary pyramid. An incision was made into the dura, and the pyramid was transected 
Figure 1. Scheme of the corticofugal projections from the primary motor cortex to the red nucleus and the basilar pontine nuclei. In addition, the corticospinal and rubrospinal tracts are shown. Both tracts project largely to the contralateral spinal cord. The arrow indicates the lesion site of the CST, caudal to the red nucleus and the pons but rostral to the decussation of the CST.

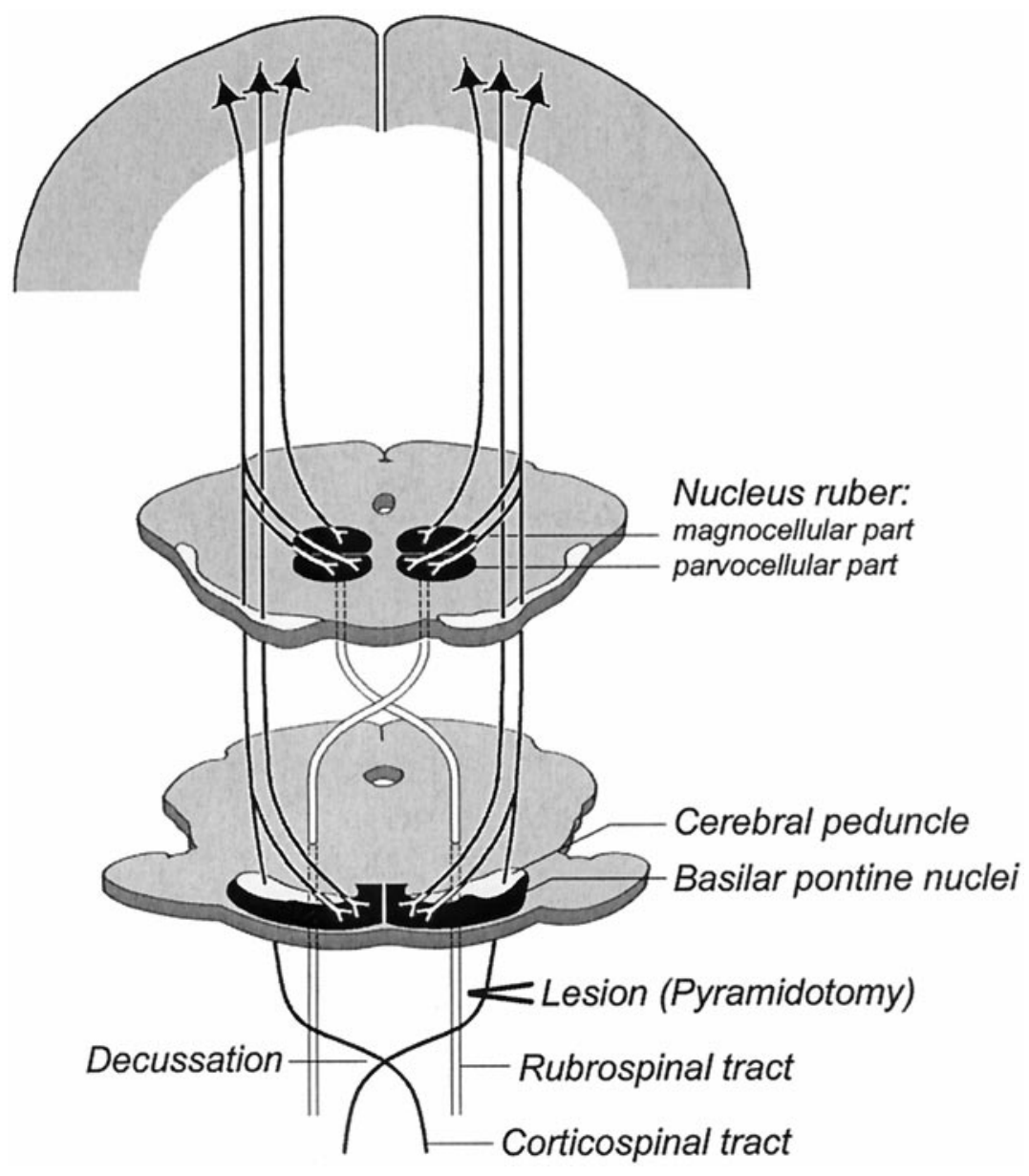

$\sim 0.5 \mathrm{~mm}$ rostral to the decussation with a sharpened \#11 scalpel blade. The wound was sutured, and the pups were warmed on a heating plate until fully awake before being returned to their mother. Pyramidotomy in adult rats (3 months of age) was performed as described earlier by Z'Graggen et al. (1998).

\section{Tracing and BDA histochemistry}

Three months after lesion of the CST, the caudal forelimb area of the primary motor cortex (Neafsey et al., 1986) was traced using pressure injections of the anterograde tracer biotin dextran amine (BDA; 10,000 molecular weight, Molecular Probes, Eugene, OR). The animals were divided into the following groups: (1) animals without lesion and only tracing $(n=6),(2)$ animals with neonatal pyramidotomy and tracing of the hemisphere corresponding to the lesioned pyramidal tract $(n=6)$, (3) animals with neonatal pyramidotomy and tracing of the hemisphere contralateral to the lesioned pyramidal tract $(n=6)$, and (4) animals with adult pyramidotomy and tracing of the hemisphere corresponding to the lesioned pyramidal tract $(n=6)$. A single stereotaxical pressure injection of $0.5 \mu \mathrm{l}$ of a $10 \%$ BDA solution in $0.01 \mathrm{M}$ phosphate buffer, $\mathrm{pH} 7.2$, was made into the mentioned area $(0.5 \mathrm{~mm}$ rostral and $2.5 \mathrm{~mm}$ lateral from Bregma at a depth of $1.5 \mathrm{~mm}$ ) using a $5 \mu$ l Hamilton syringe fitted with a glass micropipette with a $50 \mu \mathrm{m}$ opening diameter (Z'Graggen et al., 1998).

Fourteen days after BDA injection, animals were perf used transcardially with Ringer's solution containing $100,000 \mathrm{IU} / 1$ of heparin (Liquemin, Roche, Basel, Switzerland) and $0.25 \% \mathrm{NaNO}_{2}$ followed by the fixative (4\% paraformaldehyde in $0.1 \mathrm{M}$ phosphate buffer with $5 \%$ sucrose). The brain and cervical spinal cord were removed, post-fixed overnight, and then transferred to a solution of $30 \%$ sucrose in $0.1 \mathrm{M}$ phosphate buffer for $3 \mathrm{~d}$. The tissue was embedded in a matrix of polymerized gelatin-chicken albumin and immediately frozen by immersion in $-40^{\circ} \mathrm{C}$ cold isopentane. Coronal sections ( $50 \mu \mathrm{m}$ thick) of the forebrain and brainstem were cut on a freezing microtome. In the area of the tracer injection site, every second section was collected, whereas every section was collected in the region of the red nucleus, the pons, and the lesion site. The sections were processed for the BDA as described earlier (Herzog and Brösamle, 1997). Finally, the sections were lightly counterstained with cresyl violet and coverslipped with Eukitt (Kindler, Freiburg, Germany).

\section{Neuroanatomical analysis}

For all analyses the slides were coded, and the investigator was blind to the experimental groups. The examined brain areas were identified using the atlas of Paxinos and Watson (1986). In each animal the pyramidal tract lesion site was examined microscopically for location and completeness of the lesion as well as for damage to adjacent structures. Animals with incomplete lesions were excluded from the study. The corticofugal projections to the ipsilateral and contralateral red nucleus and to the ipsilateral and contralateral basilar pontine nuclei were analyzed quantitatively. In addition, the BDA tracer injection sites and the CST labeling were quantified. Statistical significance was assessed with the Kruskal-Wallis test (nonparametric test to compare multiple groups).

Quantification of the tracer injection site and the CST labeling. In all animals the BDA tracer injection site was localized on coronal sections, and the exact coordinates according to Bregma as well as the syringe penetration depth were determined and controlled. Electronic images of the cerebral injection site were acquired with a Xillix Microimager slowscan, high-resolution CCD camera attached to a Zeiss axiophot microscope using a $2.5 \times$ objective. The exact depth of the syringe penetration into the cerebral cortex and the area of tracer spread defined as the BDA positive area surrounding the syringe penetration site within the primary motor cortex were measured with the MCID-Program (M2 Analyzing Program, Imaging Research, Ontario, Canada).

The interanimal differences in the number of tracer-labeled CST fibers were assessed by counting BDA-positive axons in the cerebral peduncle at the same midpontine level ipsilateral to the injection site for each animal, as described earlier (Z'Graggen et al., 1998).

Quantification of the corticorubral and corticopontine projections. The corticorubral innervation from the caudal forelimb area of the primary motor cortex to the red nucleus contralateral to the injection site was analyzed by counting BDA-positive fibers crossing the midline ventral to the cerebral aqueduct on every section. A normalization was performed to correct for interanimal differences in tracing and section numbers as described by Z'Graggen et al. (1998) (midline crossing index). In parallel, the corticopontine projection to the contralateral basilar pontine nuclei was quantified by counting midline crossing fibers in the area of the pons on every second section. The normalization was performed as described for the red nucleus. In addition, the innervation density of the ipsilateral and contralateral basilar pontine nuclei was determined on the same sections. The ratio of the total contralateral versus ipsilateral density in percentage, as well as the ipsilateral projection density by normalization of the obtained densitometric values with the number of BDA-labeled fibers, was calculated (Z'Graggen et al., 1998). 


\section{Electrophysiology}

Three months after lesion, animals of all three groups ( $\mathrm{P} 2$ lesioned, $n=12$; adult lesioned, $n=4$; no lesion, $n=5$ ) underwent intracortical microstimulation (ICMS) of the sensorimotor cortex of both hemispheres and simultaneous electromyographic (EMG) recordings of proximal forelimb muscles to assess the evoked movements and the corresponding latency of the EMG responses.

Animals were anesthetized with ketamine $[100 \mathrm{mg} / \mathrm{kg}$ body weight, i.p.; additional doses of ketamine $(10 \mathrm{mg}$, i.m.) were given whenever necessary, depending on the reflex status of the animal determined by the movement response to tail pinch] and fixed in a stereotaxic frame. A bilateral craniotomy was made to expose the primary motor cortex of both hemispheres. During the stimulation procedure the dura was covered with mineral oil, and the cerebrospinal fluid was drained from the cannulated cisterna magna. Three to eight points in the previously described caudal forelimb area (Neafsey et al., 1986; Rouiller et al., 1993) of the primary motor cortex of both hemispheres were stimulated using tungsten microelectrodes with a resistance of $\sim 3.5 \mathrm{M} \Omega$. Stimulation was applied with a train duration of $60 \mathrm{msec}(0.2 \mathrm{msec}$ pulses, $330 \mathrm{~Hz})$ at a depth of $1.5-1.9 \mathrm{~mm}$. The stimulation points were selected according to the following criteria: (1) the observed movement had to be a forelimb movement, (2) a clear EMG response had to be evoked, and (3) the current threshold for ICMS had to be $<25 \mu \mathrm{A}$. For EMG recordings, two pairs of multistranded Tefloncoated wires (Cooner wire, AS 632) with exposed tips of 2-3 mm were inserted as electrodes into the elbow flexors and extensors of both forelimbs. Because of the small size of the rat forelimb muscles, EMG activity was related to a muscle group (elbow flexors or elbow extensors) and not to individual muscles. The position of the EMG electrodes was determined by checking the congruence between observed movement and the EMG response near the current threshold of ICMS. The EMG was amplified, filtered $(30-300 \mathrm{~Hz})$, and digitized with a sampling rate of $5 \mathrm{kHz}$ with the Digi-Data interface (Axon Instruments, Foster City, CA). For every recording, the type of movement, the laterality, the electrode depth of best response, and the threshold (i.e., lowest current that evoked a visible movement or an EMG response) were recorded. The delay of the EMG response was measured from the first stimulus pulse to the onset of EMG activity after summarization of 40-60 EMG traces. The delays of EMG responses elicited by ICMS with currents between 40 and $50 \mu \mathrm{A}$ were statistically analyzed, and the mean onset latency was calculated for each hemisphere of each animal. Statistical significance was assessed with the Kolmogoroff-Smirnoff test.

To temporarily inactivate the red nucleus, five animals lesioned at P2 underwent injections of the GABA agonist muscimol into both red nuclei. One microliter of a muscimol solution $(1 \mu \mathrm{g} / \mu \mathrm{l}$ in $0.9 \%$ saline) was stereotaxically pressure-injected into each red nucleus [coordinates: $5 \mathrm{~mm}$ caudal, $1.4 \mathrm{~mm}$ lateral to Bregma, and $7.8 \mathrm{~mm}$ deep (taking the surface of the skull as zero) using a penetration angle of $2.5^{\circ}$ to the vertical axis]. Such muscimol injections have been shown to result in a drug spread of 1.6 $\mathrm{mm}$ and a maximally reduced glucose uptake in a region of $1 \mathrm{~mm}$ around the injection site after $10 \mathrm{~min}$ (Martin, 1991). Animals underwent ICMS and EMG recording just before the muscimol injection, then 30 min after, and then $7 \mathrm{hr}$ after. The localization of the muscimol injection sites was confirmed by histological examinations.

Three animals (one animal lesioned at P2, two normal animals) were injected exactly in the same way as described above with ibotenic acid (10 $\mu \mathrm{g} / \mu \mathrm{l})$ for permanent lesion of the red nuclei. These animals underwent ICMS and EMG recording $1.5 \mathrm{~d}$ after ibotenic acid injection.

\section{RESULTS}

\section{Neuroanatomical analysis}

\section{Lesion site}

In all animals the lesion sites were located at the caudal end of the medulla oblongata ventral to the inferior olive. Only animals with a completely transected pyramidal tract and with no major damage to deeper structures such as the medial lemniscus or the inferior olive were included in this study. For these reasons, five P2 lesioned animals and two adult lesioned animals had to be excluded.

In the animals lesioned at P2, the inferior olive was found to form the ventral surface of the medulla oblongata in the area where the pyramidal tract would normally be found. In animals injected ipsilateral to the lesion with the anterograde tracer BDA, labeled fibers were seen to leave the CST stump rostral to the lesion in a dorsal direction. Some fibers crossed the midline toward the contralateral side, concentrated in the area medial to the trigeminal nucleus, and extended caudally into the spinal cord as an aberrant CST bundle, very similar to the situation in hamsters described earlier (Kalil and Reh, 1982). In our animals the number of these fibers reaching the cervical spinal cord was very low. No axons were seen to regenerate through the lesion nor to extend on the normal pathway through the pyramidal decussation.
In animals lesioned as adults, the area of the lesion was filled with scar tissue, and the lesioned CST fibers were retracted rostrally from the lesion site and formed retraction bulbs. In agreement with earlier observations (Raineteau et al., 1999), almost no local sprouting reaction and no regeneration could be observed in these animals.

\section{$B D A$ injection site}

All animals showed injections centered in the caudal forelimb area of the primary motor cortex (Neafsey et al., 1986) without tracer spread into the deep white matter or subcortical structures. The mean tracer spread measured on cross sections of the primary motor cortex at the site of syringe penetration was $3.84 \times 10^{6} \mu \mathrm{m}^{2}$ $\left( \pm 0.22 \times 10^{6} \mathrm{SEM}, n=24\right)$. No differences were found among the animal groups. Minor colabeling of neighboring face and hindlimb areas of the primary motor cortex could not be excluded.

The analysis of the cerebral peduncle at midpontine level showed a typical and consistent distribution of BDA-positive fibers within the peduncle on cross sections. For all animal groups, most of the BDA-positive fibers were situated in the medial half of the cerebral peduncle, whereas only a few were seen in the lateral half, as described earlier for the projections from the caudal forelimb area of the motor cortex (Mihailoff et al., 1978; Kosinski et al., 1986). The number of labeled fibers within the cerebral peduncle did not vary significantly among the groups, although fewer BDAlabeled fibers tended to be in the animals lesioned at P2, for both the lesioned as well as the unlesioned CST (6181 \pm 1068 SEM, $n=$ 6 , for the lesioned CST and $4628 \pm 433$ SEM, $n=6$, for the unlesioned CST), compared with the adult lesioned $(8075 \pm 1548$ SEM, $n=6)$ or unlesioned animals $(7029 \pm 583$ SEM, $n=6)$. This lower CST axon number may be attributable to some cell death occurring after newborn lesions (Tolbert and Der, 1987; Merline and Kalil, 1990).

\section{Corticorubral projection}

The corticorubral projection on the side of the lesion was identical in all of the experimental groups. Labeled fibers originating from the caudal forelimb area of the primary motor cortex were found to descend to midbrain levels either via the cerebral peduncle or through the thalamus. They innervated mainly the parvocellular part of the ipsilateral red nucleus, the pararubral area, the prerubral field, and the region around the fasciculus retroflexus, as described earlier (Brown, 1974; Gwyn and Flumerfelt, 1974; Flumerfelt, 1980; Naus et al., 1985a,b). A few fibers terminated in the magnocellular portion of the ipsilateral red nucleus with bouton-like endings.

In unlesioned rats and animals lesioned as adults, only a few fibers crossed the midline and terminated mainly in the parvocellular region of the contralateral red nucleus. The sum of midline crossing fibers $(300-400)$ related to the total number of labeled CST fibers for each rat to correct for the differences in the tracing and multiplied by $10^{4}$ gave a midline fiber crossing index of $416 \pm$ $46(\mathrm{SEM}, n=6)$ for unlesioned animals and of $509 \pm 87(n=6)$ for animals lesioned as adults (not statistically different). Most of these fibers crossed in the rostral two-thirds of the red nucleus.

In animals that underwent a lesion at P2 and BDA labeling of the hemisphere corresponding to the lesioned pyramidal tract, the proportion of corticorubral fibers crossing the midline and terminating in the contralateral red nucleus was greatly increased [crossing fiber index of $1718 \pm 176(n=6), p<0.01$ ] (Figs. $2 A, B, 3 A, B)$. Some of these crossing fibers could be identified as branches of ispsilateral corticorubral axons. Some of these fibers directly crossed the midline toward the contralateral red nucleus, whereas others crossed more dorsally in the central gray (Fig. $2 A, B$ ). The crossing axons terminated mainly in the parvocellular part of the contralateral red nucleus with bouton-like endings; a few axons were seen to end in the magnocellular part.

In animals with lesion at P2 and BDA tracing of the hemisphere corresponding to the intact CST, a decrease of the crossing fiber index was found compared with all the other experimental animal 

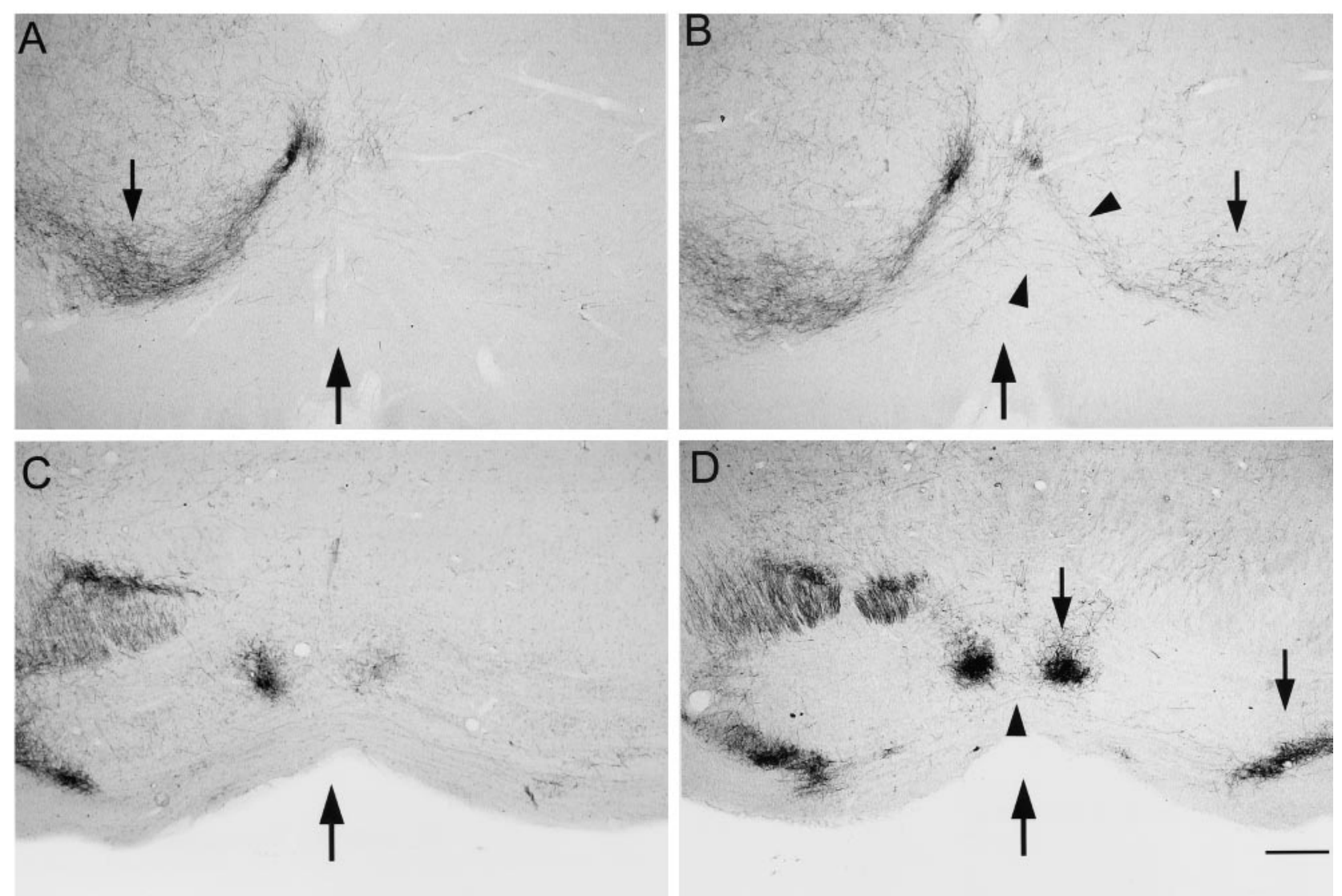

Figure 2. $A, B$, Cross sections of the nucleus ruber. $A$, Corticofugal projection to the red nucleus of an animal lesioned in the pyramid (PTX) as adult. As in unlesioned controls, the fibers terminate almost exclusively in the ipsilateral red nucleus (small arrow); very few fibers crossing the midline are present. B. Animal with PTX at P2. Many axons (arrowheads) cross the midline and terminate in the area of the contralateral red nucleus (arrow). $C$, $D$, Cross sections at midpontine level. $C$, Animal lesioned as adult. Labeled fibers leave the cerebral peduncle and form the ipsilateral forelimb-specific termination zones, almost completely restricted to the ipsilateral basilar pontine nuclei. Very few fibers end on the contralateral side close to the midline. $D$, Animal lesioned at P2. A large increase in the number of midline crossing fibers (arrowhead) and in the innervation of the contralateral basilar pontine nuclei can be observed (small arrows), whereas the ipsilateral side seems unchanged. The contralateral termination fields mirror the ipsilateral ones. Scale bar, $280 \mu \mathrm{m}$. Magnification 35×. Large arrow indicates midline.

\section{Nucleus Ruber: midline crossing fibers}
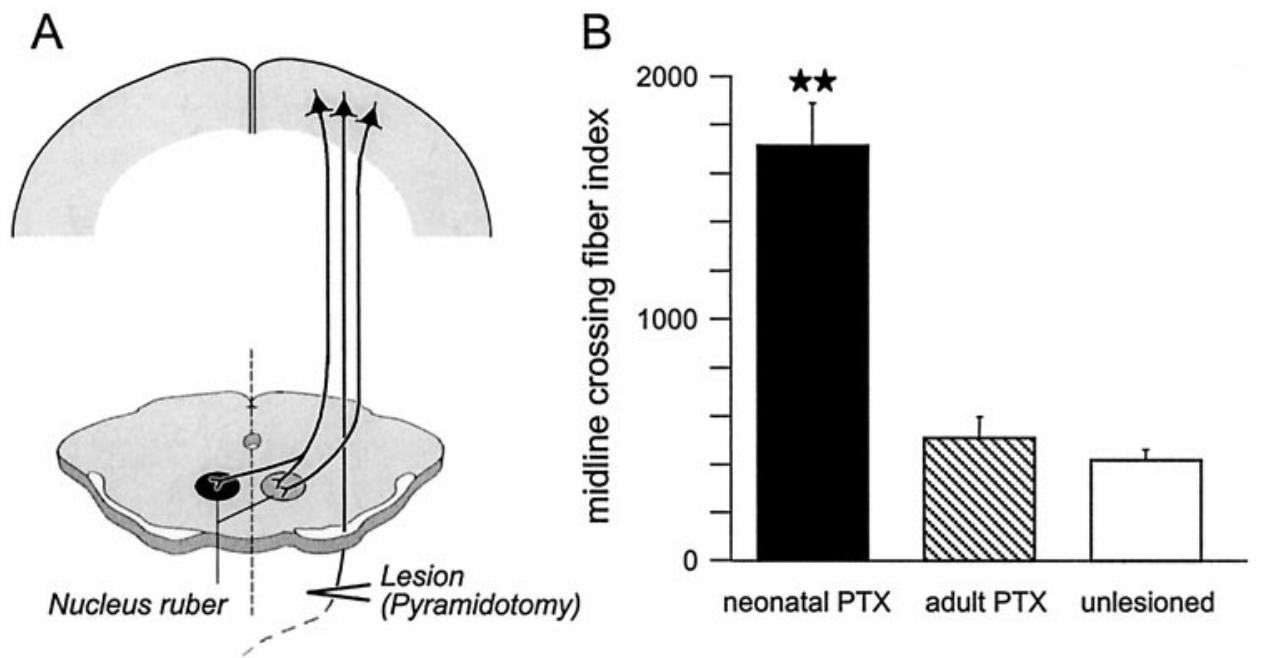

Figure 3. A, Scheme of the projection from the primary motor cortex corresponding to the lesioned CST (arrowhead) to the ipsilateral and contralateral red nucleus. The dashed line indicates the midline. $B$, Midline fiber crossing index: number of midline crossing fibers in the area of the red nucleus divided by the total number of labeled CST fibers (to correct for the differences in the tracing) and multiplied by $10^{4}$. Error bars indicate SEM. ${ }_{* *}^{*} p<0.01$; Kruskal-Wallis test. 


\section{Cortico - pontine projection}
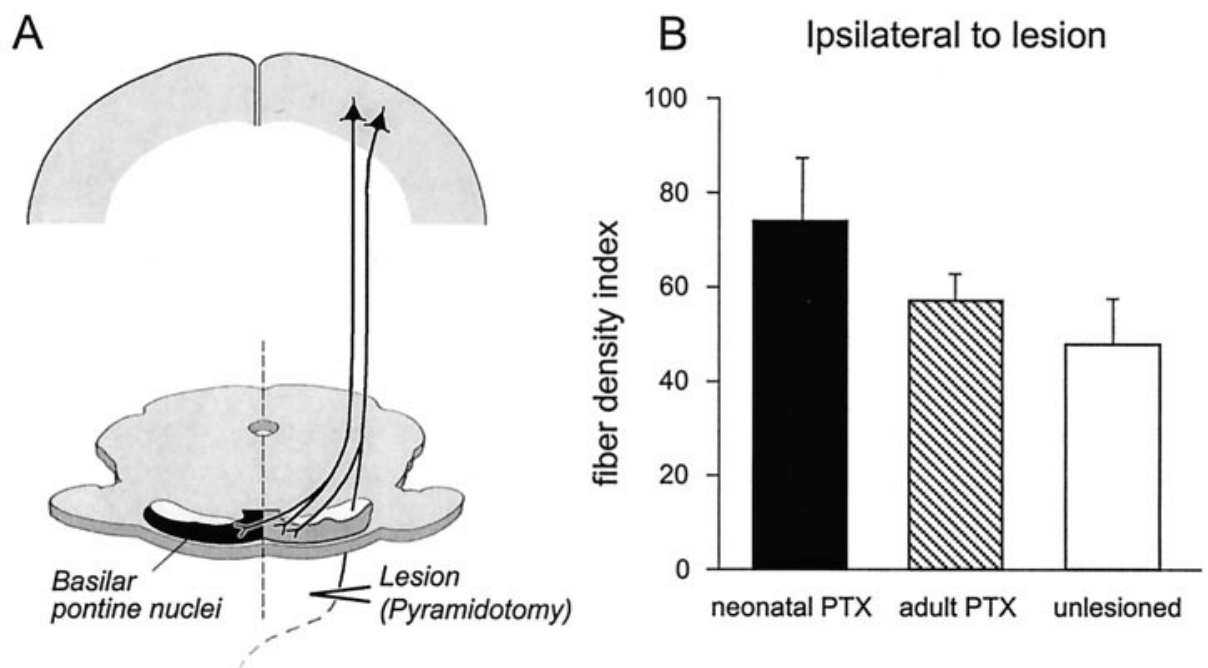

\section{Contralateral to lesion}

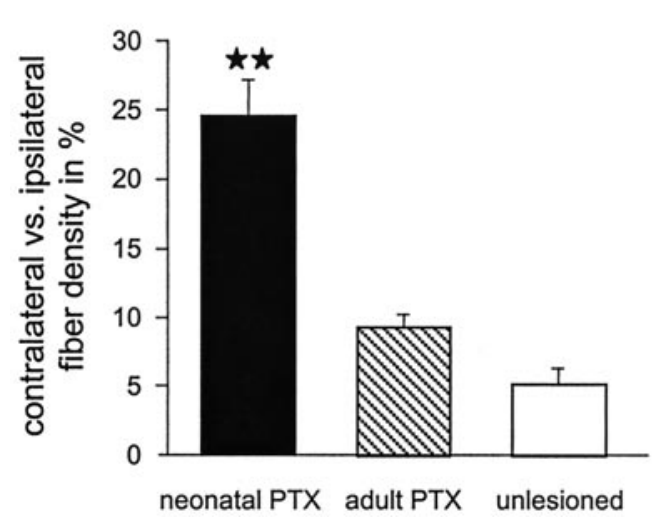

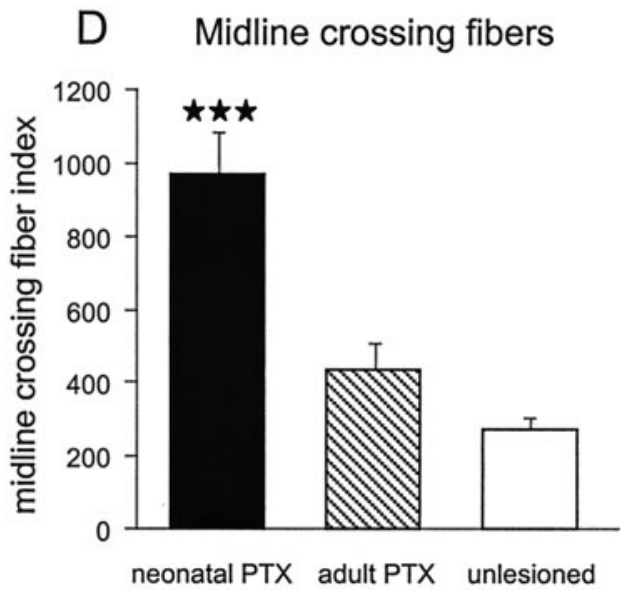

Figure 4. A, Scheme of the projection from the primary motor cortex corresponding to the lesioned CST (arrowhead) to the ipsilateral and contralateral basilar pontine nuclei. The dashed line indicates the midline. $B$, Fiber density in the ipsilateral corticopontine innervation fields, divided by the number of labeled fibers. $C$, Density of the terminal fields of corticopontine projections to the contralateral basilar pontine nuclei, expressed as percentage of the ipsilateral innervation density. ${ }^{* *} p<0.01$; KruskalWallis test. $D$, Midline crossing fiber index in the area of the basilar pontine nuclei (number of midline crossing fibers in the area of the pons divided by the total number of labeled CST fibers and multiplied by $\left.10^{4}\right)$. ${ }^{* * *} p<0.01$; KruskalWallis test. Error bars indicate SEM. groups $(267 \pm 39, n=6$; decrease by $36 \%$ compared with unlesioned animals).

\section{Corticopontine projection}

The cortical projection from the hemicortex corresponding to the lesioned CST to the ipsilateral basilar pontine nuclei showed the typical topographical forelimb-specific innervation pattern in all experimental groups (Mihailoff et al., 1978; Wiesendanger and Wiesendanger, 1982; Rouiller et al., 1993; Panto et al., 1995). At rostral levels one central termination field was observed that divided into a medial, ventral, and lateral column at midpontine levels. In addition, a new termination zone dorsal to the cerebral peduncle appeared. Further caudally, the three columns merged into one termination field covering the medial, ventral, and lateral pons and the area around the medial and dorsal aspect of the cerebral peduncle. In normal unlesioned rats, a very minor projection to the contralateral pons, located mainly at mid- to caudal levels could always be observed.

The analysis of the corticopontine projection on the side of the lesion showed no significant differences between the experimental groups, although in animals lesioned at P2 a slightly higher fiber density could be seen (Fig. $4 B$ ). In contrast, a marked increase in the innervation of the pontine nuclei on the side opposite to the lesion was found in the P2 lesioned rats (Fig. 2C,D, 4C). Densitometric analysis showed the contralateral fiber density to be $24.6 \pm$ $2.6 \%(n=6)$ of the ipsilateral innervation density in the animals lesioned at $\mathrm{P} 2$, as compared with $9.3 \pm 0.9 \%(n=6)$ in the animals lesioned as adults and $5.2 \pm 1.1 \%(n=6)$ in unlesioned animals. The small increase in the adult lesioned animals compared with the unlesioned animals was not statistically significant. The enhanced contralateral projection formed terminal fields mirroring exactly the ipsilateral innervation pattern at the same level, thus showing a topographically correct, forelimb-specific projection to the opposite basilar pontine nuclei.

The enhanced contralateral projection in animals lesioned at P2 was also reflected by a highly significant increase $(p<0.001)$ in the midline crossing fiber index (number of midline crossing fibers in the area of the pons divided by the total number of labeled CST fibers multiplied by $\left.10^{4}\right)$ : $968 \pm 109(n=6)$ in animals lesioned at P2 compared with $436 \pm 70(n=6)$ in the animals lesioned as adults and $271 \pm 30.6(n=6)$ in unlesioned animals (Fig. $4 D)$. No significant difference could be observed between the rats lesioned as adults and unlesioned animals (Z'Graggen et al., 1998).

In animals with lesion at P2 and BDA tracing of the hemisphere corresponding to the intact CST, a decrease of the crossing fiber index $(175 \pm 25, n=6)$ was found compared with all other animal groups, including the unlesioned animals. With regard to the contralateral termination density (in percentage of the ipsilateral density), these animals were not distinguishable from unlesioned animals and rats lesioned as adults $(5.98 \pm 1.2, n=6)$.

These anatomical data show a marked increase in the number of corticorubral and corticopontine fibers from the lesioned side crossing the midline and an increase in the density of the contralateral terminal plexus in response to neonatal pyramidotomy. 


\section{Electrophysiology}

In normal unlesioned animals, ICMS of the caudal forelimb area of the primary motor cortex elicited movements in the contralateral elbow or wrist, or both. Best responses were found at a stimulation electrode penetration depth between 1.6 and $1.8 \mathrm{~mm}$. The mean stimulation current threshold intensity in normal animals was $19.5 \pm 0.45 \mu \mathrm{A}(n=3)$, and the onset of the EMG response for the contralateral elbow flexors was $13.8 \pm 0.6 \mathrm{msec}$ (Fig. $5 A, B$ ). In $\sim 35 \%$ of the stimulation points bilateral movements were elicited. The onset latency of the ipsilateral elbow flexor EMG was significantly delayed compared with the contralateral side $(26.8 \pm 0.62$ msec) (Fig. 5A, $B$ ). In most of these stimulation sites with bilateral responses, the ipsilateral component showed a tendency for a higher threshold compared with the contralateral movement component, as described earlier (Kartje-Tillotson et al., 1985; Liang et al., 1993). These findings were similar for both hemicortices.

After pyramidotomy in adult rats, ICMS of the caudal forelimb motor area on the lesioned side was ineffective in evoking any muscle responses at currents below $80 \mu \mathrm{A}$ (Fig. 5B). Stimulation of the caudal forelimb motor area of the intact side resulted in EMG latencies for the contralateral elbow flexors that were comparable with those in unlesioned animals $(13.25 \pm 2.25 \mathrm{msec})$ (Fig. 5B).

A different situation was found in animals lesioned at P2. In these animals, stimulation of the caudal forelimb motor cortex on the lesioned side resulted in bilateral forelimb movements. The onset latencies of these EMG responses were significantly shorter for the ipsilateral $(25.20 \pm 2.22 \mathrm{msec}, n=11)$ than for the contralateral elbow flexors (33.22 $\pm 2.39 \mathrm{msec}, n=9, p<0.05)$ (Fig. $5 A, B$ ). Both EMG latencies were significantly prolonged as compared with the EMG latency measured for the contralateral elbow flexors in unlesioned animals. ICMS of the caudal forelimb motor cortex on the intact side resulted almost exclusively in contralateral forelimb movements with a mean latency of $16.79 \pm 1.16 \mathrm{msec}$ $(n=11)$ (Fig. $5 B)$. This value was identical to that of unlesioned animals (Fig. $5 B$ ).

To investigate the possible role of a rearranged corticorubral projection in the $\mathrm{P} 2$ lesioned animals, the red nuclei were inactivated by local injections of the GABA receptor agonist muscimol $(n=5)$. In two of these animals, both red nuclei were injected with muscimol $30 \mathrm{~min}$ before cortical stimulation. Stimulation of the motor cortex of the lesioned side with currents up to $75 \mu \mathrm{A}$ did not evoke any EMG response in these rats (Fig. $5 C$ ). In three rats the nucleus ruber on the lesioned side was injected first with muscimol. Stimulation of the forelimb motor cortex of the lesioned side 30 min later resulted in a complete loss of the contralateral movement in all of the animals, whereas the ipsilateral flexor EMG latencies remained unaffected. This ipsilateral movement component disappeared completely, however, after the injection of muscimol into the other red nucleus. In contrast to these results seen by ICMS of the lesioned side cortex, stimulation of the opposite forelimb motor cortex in all of these animals resulted in normal short-latency EMGs that were unaffected by the muscimol injections (Fig. 5C). Seven hours after the first muscimol injection, restimulation of the motor cortex on the lesioned side showed recovery of the ipsilateral as well as the contralateral forelimb EMG responses (Fig. 5C). The prolonged latencies could be caused by unspecific inhibitory effects of the muscimol, in line with a deeper anesthesia level of the animals observed after the muscimol injections.

Histological analysis of the red nuclei of all animals with muscimol injections showed that all injections were centered within the nucleus; in all animals a small area of tissue necrosis could be observed (data not shown).

To confirm the results above, three rats (one rat lesioned at P2 and two normal rats) were injected with ibotenic acid into both red nuclei for permanent lesions. ICMS was performed $1.5 \mathrm{~d}$ after the injections of ibotenic acid. The results obtained by stimulation of the animal lesioned at P2 were similar to the ones described after bilateral injection of muscimol: no movements could be evoked by stimulation of the motor cortex on the lesioned side, whereas the responses to stimulation of the intact motor cortex were identical to those of normal animals. The histological analysis showed a complete lesion of both red nuclei. The two normal control animals with similar ibotenic acid lesions of both red nuclei showed exactly the same EMG latencies for the contralateral elbow flexors as normal animals. The ipsilateral movement component was also unchanged in these animals. In contrast, stimulation of a normal adult animal before and after injections of muscimol into the ipsilateral and contralateral red nucleus resulted in a slight slowdown of EMG latencies compared with the latencies obtained before muscimol injections.

\section{DISCUSSION}

This study shows that neonatal unilateral lesion of the CST led to specific corticorubral and corticopontine plasticity: the motor cortex that has lost its direct access to the spinal cord via the CST established a bilateral innervation of the red nucleus and the basilar pontine nuclei. Cortical microstimulation showed that this motor cortex was reconnected to the periphery. The transient inactivation of the red nucleus with muscimol resulted in a complete loss of these cortically evoked EMGs, suggesting that a cortico-rubrospinal connection substituted for the loss of the direct corticospinal pathway.

\section{Neonatal unilateral pyramidotomy enhances corticorubral and corticopontine plasticity}

Our results demonstrate an increased number of corticorubral and corticopontine fibers crossing the midline and innervating the contralateral red nucleus and basilar pontine nuclei as a consequence of neonatal unilateral pyramidotomy. In the red nucleus, these crossing fibers mainly ended in the contralateral parvocellular region, i.e., in the normal main target site for corticorubral fibers (Brown, 1974; Gwyn and Flumerfelt, 1974; Flumerfelt, 1980; Naus et al., 1985a,b). A few fibers also terminated in the magnocellular part of the red nucleus ipsilaterally and contralaterally. In the pons the enhanced contralateral innervation mirrored exactly the ipsilateral termination pattern (Mihailoff et al., 1978; Wiesendanger and Wiesendanger, 1982; Panto et al., 1995). The fiber density on the intact side and its somatotopic termination pattern were unchanged after neonatal lesion compared with the control groups. Very similar changes in connectivity of corticobulbar fibers were observed after neonatal cortical lesions: the remaining cortex established a bilateral projection to the red nuclei and the pons by an increased number of midline crossing fibers (Leong and Lund, 1973; Nah and Leong, 1976a,b; Castro and Mihailoff, 1983; Naus et al., 1985a,b; Kartje-Tillotson et al., 1986; Murakami et al., 1990, 1993).

\section{The primary motor cortex that has lost its connection to the spinal cord obtains new functional access through the nucleus ruber}

After neonatal pyramidotomy, ICMS of the forelimb motor cortex corresponding to the lesioned CST resulted in bilateral forelimb movements. EMG latencies were longer for the ipsilateral as well as the contralateral movements compared with EMG latencies evoked by stimulation of the intact hemicortex in these animals or in normal unlesioned animals. In contrast to these findings in newborn lesioned rats, low-threshold ICMS after adult pyramidotomy could not evoke any forelimb motor responses, as described earlier for the monkey (Mitz and Humphrey, 1986) and the rat (KartjeTillotson et al., 1987).

These findings demonstrate that after neonatal pyramidotomy, the cortex, which was no longer connected to the periphery via the CST, became reconnected. The prolonged EMG latencies and the bilateral movement pattern suggest the involvement of indirect pathways. Because our anatomical results after P2 lesion showed a bilateral corticorubral projection, we investigated whether this new pathway could mediate the forelimb movements. Indeed, transient inactivation of both red nuclei with the GABA receptor agonist 
A

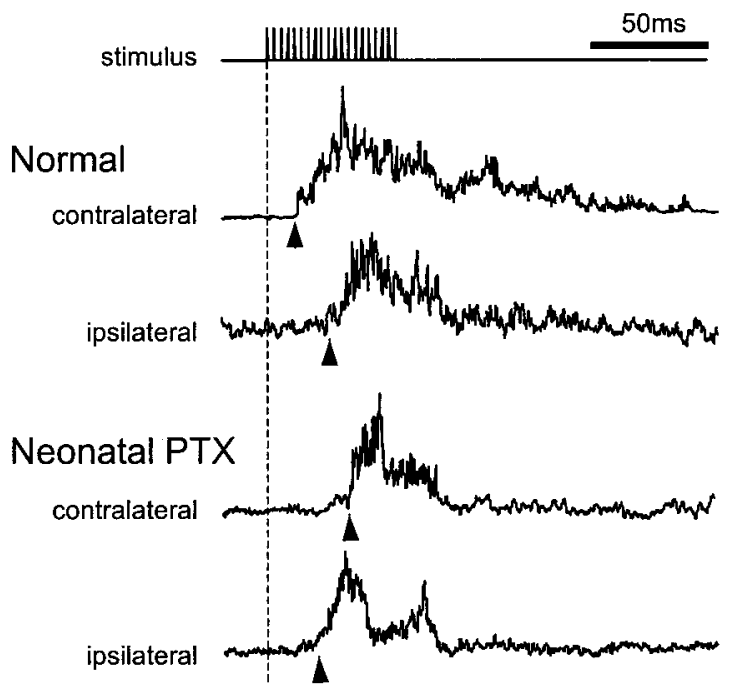

B
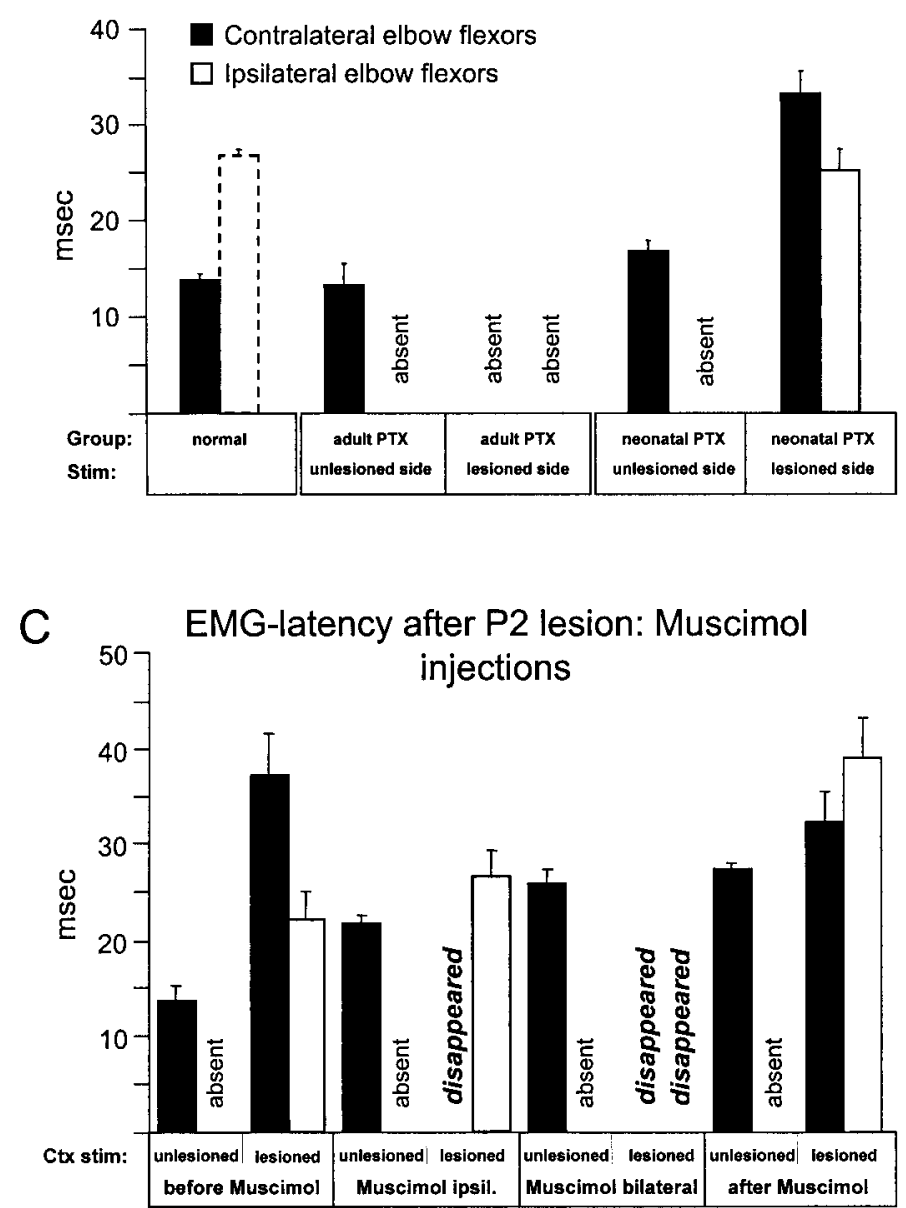

Figure 5. A, Rectified and averaged EMG recordings (elbow flexors) of 50 single traces elicited by intracortical microstimulation (ICMS). ICMS in normal unlesioned rats resulted in a fast contralateral and in $\sim 35 \%$ of the stimulations also in an ipsilateral response. Stimulation of the hemisphere corresponding to the lesioned pyramidal tract after neonatal lesion always elicited bilateral movements. $B$, EMG latencies of the elbow flexors elicited by ICMS of the forelimb motor cortex on the lesioned or unlesioned side. ICMS in a normal animal resulted in fast contralateral responses; in $\sim 35 \%$ of the stimulations an ipsilateral EMG with a prolonged delay could also be found (dashed line column). ICMS of the cortex of the intact side in animals after adult or neonatal pyramidotomy resulted in normal contralateral movements with short EMG delays. In rats lesioned at P2, stimulation of the muscimol in animals lesioned at $\mathrm{P} 2$ resulted in a complete loss of the forelimb EMGs after stimulation of the motor cortex of the lesioned side. Responses of the intact side were unaffected by the inactivation of the red nucleus. Inactivation or lesion of the red nucleus with muscimol or ibotenic acid had no effect in normal unlesioned control animals. This shows that the nucleus ruber is not mediating the early and main phase of the EMG evoked by ICMS in normal animals. The results in P2 lesioned rats strongly suggest that the red nucleus becomes a crucial structure in a new pathway from the cortex of the lesioned side to the spinal cord. This pathway could induce a direct activation of the rubrospinal tract (RST). The RST is a mainly crossed pathway originating from the magnocellular part of the red nucleus (Antal et al., 1992). We could detect labeled corticorubral fibers ending in the ipsilateral and contralateral magnocellular part of the red nucleus after neonatal lesion. Furthermore, the CST and RST have many functional similarities (Kennedy, 1990) and overlapping target fields in the spinal cord (for review, see Jankowska, 1988). Therefore, a possible compensatory takeover of CST function by the RST after a CST lesion could be possible. A second explanation for our findings would be a more complex pathway originating, e.g., in the parvocellular region of the red nucleus and including the cerebellar loop. The observed corticopontine plasticity could also play a role in this context. The basilar pontine nuclei are an important relay in the connection of the cerebral cortex and the cerebellum. It can be assumed that a lesion of the CST induces not only a change in the connectivity to the spinal cord but also of other motor pathways, such as the cerebellar loop.

The possible role of regenerated CST fibers in our rats is unclear. Kalil and Reh (1982) demonstrated in hamster a growth of CST fibers into the spinal cord after neonatal pyramidotomy, along with a high degree of recovery of forelimb and hand function (Reh and Kalil, 1982). Our electrophysiological experiments with muscimol suggest that in our study these fibers are not responsible for the low-threshold forelimb EMGs elicited by ICMS. Therefore, the possibility exists that in the hamster experiments, plastic rearrangement of connections like the ones described here could also have contributed to the functional recovery observed.

\section{Underlying mechanisms}

The formation of important bilateral corticobulbar projections was also described after cortical lesions in neonatal rats, where the spared, unablated cortex was shown to send bilateral projections to the nucleus ruber (Leong and Lund, 1973; Nah and Leong, 1976a,b; Naus et al., 1985a,b) and the basilar pontine nuclei (Leong and Lund, 1973; Castro and Mihailoff, 1983; Kartje-Tillotson et al., 1986). These cortical lesions result in a unilateral deafferentation of the corresponding red nucleus and pons, a process that could induce signals that promote sprouting. Pyramidotomy, however, leaves the corticorubral and corticopontine innervation of both sides intact. Thus, growth-promoting factors, survival factors, and attracting guidance factors may be upregulated because of functional imbalances existing in these motor circuits as a consequence of the pyramidal lesion.

$\leftarrow$

motor cortex of the lesioned side always evoked bilateral responses with significantly prolonged delays $(p<0.05)$. In rats lesioned as adults, no EMGs could be evoked by ICMS of the lesion-side cortex at low current intensities. $C$, EMG latencies in animals lesioned at P2 before and after temporary inactivation of the ipsilateral or of both red nuclei with muscimol. Muscimol injections into the red nucleus ipsilateral to the lesion lead to a loss of the contralateral EMGs. After the additional inactivation of the opposite red nucleus with muscimol, no EMGs could be evoked by ICMS. Stimulation of the unlesioned side after muscimol injections (one or both red nuclei) did not affect the occurrence of contralateral EMGs. The prolonged latencies are probably caused by unspecific inhibitory effects of muscimol. Seven hours after muscimol injection, ICMS on the lesioned side resulted again in bilateral movements. The prolonged EMG latency for the ipsilateral elbow flexors compared with values before muscimol injections, in contrast to the full recovery found for the contralateral elbow flexors, could be explained by the shorter $(5.5 \mathrm{hr})$ recovery time after muscimol injection of the contralateral red nucleus. 
The increase in the number of midline crossing fibers in the red nucleus and the pons after neonatal pyramidotomy described here could be the result of lesion-induced sprouting across the midline toward the contralateral red nucleus and basilar pontine nuclei, or of a lesion-induced stabilization of early, transient bilateral cortical projections (Murakami et al., 1990, 1993). Factors attracting cortical fiber collaterals, e.g., into the pons, are expressed there at late embryonic stages to establish the normal innervation of the pontine nuclei (Heffner et al., 1990; O'Leary et al., 1991). Such factors could also serve to attract fibers from the opposite side of the pons or to enhance elaboration of a terminal plexus.

Although most of the cortical fibers ending in the red nucleus and the pons are true corticorubral and corticopontine fibers, some of these projections are collaterals of CST fibers (Ugolini and Kuypers, 1986; Akintunde and Buxton, 1992). Transection of the CST could therefore also induce collateral sprouting in the sense of a "pruning effect" (Sabel and Schneider, 1988). The present results do not allow us to distinguish between these possibilities.

The capacity for plasticity and regeneration of the CST decreases greatly during postnatal development (Kuang and Kalil, 1990; Firkins et al., 1993), a process that coincides in time with the formation of myelin (Kapf hammer and Schwab, 1994). Neutralization of myelin-associated neurite growth inhibitors with the monoclonal antibody IN-1 resulted in enhanced regeneration of CST axons after spinal cord lesion (Schnell and Schwab, 1990, 1993; Bregman et al., 1995). Interestingly, treatment with the antibody IN-1 after selective unilateral pyramidotomy in adult rats induced sprouting of corticorubral and corticopontine fibers, resulting in an enhanced bilateral innervation of anatomically correct parts of the red nucleus and the basilar pons, very similar to the situation observed here in newborn lesioned animals (Z'Graggen et al., 1998). In parallel, a high degree of functional recovery in skilled forelimb movements and grip strength occurred (Z'Graggen et al., 1998). Thus, neutralization of myelin-associated neurite growth inhibitor activity by the antibody IN-1 in adult animals induced sprouting and changes in the corticofugal connectivity that can occur spontaneously only in the newborn brain. In animals undergoing lesion at birth, an overlap between development and lesioninduced reorganization probably takes place. Interestingly, although the conditions are different after neonatal and adult lesions, the CNS seems to react in a similar way, leading to a high level of functional recovery in both cases.

\section{REFERENCES}

Akintunde A, Buxton DF (1992) Origins and collateralization of corticospinal, corticopontine, corticorubral and corticostriatal tracts: a multiple retrograde fluorescent tracing study. Brain Res 586:208-218.

Antal M, Sholomenko GN, Moschovakis AK, Storm-Mathisen J, Heizmann CW, Hunziker W (1992) The termination pattern and postsynaptic targets of rubrospinal fibers in the rat spinal cord: a light and electron microscopic study. J Comp Neurol 325:22-37.

Bregman BS, Kunkel-Bagden E, Schnell L, Dai HN, Gao D, Schwab ME (1995) Recovery from spinal cord injury mediated by antibodies to neurite growth inhibitors. Nature 378:498-501.

Brown LT (1974) Corticorubral projections in the rat. J Comp Neurol 154:149-167.

Castro AJ (1975) Ipsilateral corticospinal projections after large lesions of the cerebral hemisphere in neonatal rats. Exp Neurol 46:1-8.

Castro AJ, Mihailoff GA (1983) Corticopontine remodelling after cortical and/or cerebellar lesions in newborn rats. J Comp Neurol 219:112-123.

Donoghue JP (1995) Plasticity of adult sensorimotor representations. Curr Opin Neurobiol 5:749-754.

Donoghue JP (1997) Limits of reorganization in cortical circuits. Cereb Cortex 7:97-99.

Firkins SS, Bates CA, Stelzner DJ (1993) Corticospinal tract plasticity and astroglial reactivity after cervical spinal injury in the postnatal rat. Exp Neurol 120:1-15.

Flumerfelt BA (1980) An ultrastructural investigation of afferent connections of the red nucleus in the rat. J Anat 131:621-633.

Gwyn DG, Flumerfelt BA (1974) A comparison of the distribution of cortical and cerebellar afferents in the red nucleus of the rat. Brain Res 69:130-135.
Heffner CD, Lumsden AG, O'Leary DD (1990) Target control of collateral extension and directional axon growth in the mammalian brain. Science 247:217-220.

Herzog A, Brösamle C (1997) "Semifree-floating" treatment: a simple and fast method to process consecutive sections for immunohistochemistry and neuronal tracing. J Neurosci Methods 72:57-63.

Jankowska E (1988) Target cells of rubrospinal tract fibers within the lumbar spinal cord. Behav Brain Res 28:91-96.

Kalil K, Reh T (1982) A light and electron microscopic study of regrowing pyramidal tract fibers. J Comp Neurol 211:265-275.

Kapfhammer JP, Schwab ME (1994) Inverse patterns of myelination and GAP-43 expression in the adult CNS: neurite growth inhibitors as regulators of neuronal plasticity? J Comp Neurol 340:194-206.

Kartje-Tillotson G, Neafsey EJ, Castro AJ (1985) Electrophysiological analysis of motor cortical plasticity after cortical lesions in newborn rats. Brain Res 332:103-111.

Kartje-Tillotson G, Neafsey EJ, Castro AJ (1986) Topography of corticopontine remodelling after cortical lesions in newborn rats. J Comp Neurol 250:206-214.

Kartje-Tillotson G, O'Donoghue DL, Dauzvardis MF, Castro AJ (1987) Pyramidotomy abolishes the abnormal movements evoked by intracortical microstimulation in adult rats that sustained neonatal cortical lesions. Brain Res 415:172-177.

Keifer J, Kalil K (1991) Effects of infant versus adult pyramidal tract lesions on locomotor behavior in hamsters. Exp Neurol 111:98-105.

Kennedy PR (1990) Corticospinal, rubrospinal and rubro-olivary projections: a unifying hypothesis. Trends Neurosci 13:474-479.

Kosinski RJ, Neafsey EJ, Castro AJ (1986) A comparative topographical analysis of dorsal column nuclear and cerebral cortical projections to the basilar pontine gray in rats. J Comp Neurol 244:163-173.

Kuang RZ, Kalil K (1990) Specificity of corticospinal axon arbors sprouting into denervated contralateral spinal cord. J Comp Neurol 302:461-472.

Leong SK, Lund RD (1973) Anomalous bilateral corticof ugal pathways in albino rats after neonatal lesions. Brain Res 62:218-221.

Liang F, Rouiller EM, Wiesendanger M (1993) Modulation of sustained electromyographic activity by single intracortical microstimuli: comparison of two forelimb motor cortical areas of the rat. Somatosens Mot Res 10:51-61.

Martin JH (1991) Autoradiographic estimation of the extent of reversible inactivation produced by microinjection of lidocaine and muscimol in the rat. Neurosci Lett 127:160-164.

Merline M, Kalil K (1990) Cell death of corticospinal neurons is induced by axotomy before but not after innervation of spinal targets. J Comp Neurol 296:506-516.

Mihailoff GA, Burne RA, Woodward DJ (1978) Projections of the sensorimotor cortex to the basilar pontine nuclei in the rat: an autoradiographic study. Brain Res 145:347-354.

Mitz AR, Humphrey DR (1986) Intracortical stimulation in pyramidotomized monkeys. Neurosci Lett 64:59-64.

Murakami F, Song W-J, Higashi S (1990) Formation of crossed and uncrossed projections in the central nervous system. Neurosci Res [Suppl] 13:37-42.

Murakami F, Kobayashi Y, Uratani T, Tamada A (1993) Individual corticorubral neurons project bilaterally during postnatal development and following early contralateral cortical lesions. Exp Brain Res 96:181-193.

Nah SH, Leong SK (1976a) Bilateral corticofugal projection to the red nucleus after neonatal lesions in the albino rat. Brain Res 107:433-436.

Nah SH, Leong SK (1976b) An ultrastructural study of the anomalous corticorubral projection following neonatal lesions in the albino rat. Brain Res 111:162-166.

Naus C, Flumerfelt BA, Hrycyshyn AW (1985a) An anterograde HRPWGA study of aberrant corticorubral projections following neonatal lesions of the rat sensorimotor cortex. Exp Brain Res 59:365-371.

Naus CG, Flumerfelt BA, Hrycyshyn AW (1985b) An HRP-TMB ultrastructural study of rubral afferents in the rat. J Comp Neurol 239:453-465.

Neafsey EJ, Bold EL, Haas G, Hurley-Gius KM, Quirk G, Sievert CF, Terreberry RR (1986) The organization of the rat motor cortex: a microstimulation mapping study. Brain Res 396:77-96.

O'Leary DD, Heffner CD, Kutka L, Lopez-Mascaraque L, Missias A, Reinoso BS (1991) A target-derived chemoattractant controls the development of the corticopontine projection by a novel mechanism of axon targeting. Development [Suppl] 2:123-130.

Panto MR, Cicirata F, Angaut P, Parenti R, Serapide F (1995) The projection from the primary motor and somatic sensory cortex to the basilar pontine nuclei. A detailed electrophysiological and anatomical study in the rat. J Hirnforsch 36:7-19.

Paxinos G, Watson C (1986) The rat brain in stereotaxic coordinates, Ed 2. New York: Academic.

Raineteau O, Z'Graggen WJ, Thallmair M, Schwab ME (1999) Sprouting and regeneration after pyramidotomy and blockade of the myelinassociated neurite growth inhibitors NI 35/250 in adult rats. Eur J Neurosci 11:1486-1490.

Reh T, Kalil K (1982) Functional role of regrowing pyramidal tract fibers. J Comp Neurol 211:276-283.

Rouiller EM, Liang FY, Moret V, Wiesendanger M (1991) Trajectory of 
redirected corticospinal axons after unilateral lesion of the sensorimotor cortex in neonatal rat; a phaseolus vulgaris-leucoagglutinin (PHA-L) tracing study. Exp Neurol 114:53-65.

Rouiller EM, Moret V, Liang F (1993) Comparison of the connectional properties of the two forelimb areas of the rat sensorimotor cortex: support for the presence of a premotor or supplementary motor cortical area. Somatosens Mot Res 10:269-289.

Sabel BA, Schneider GE (1988) The principle of "conservation of total axonal arborizations": massive compensatory sprouting in the hamster subcortical visual system after early tectal lesions. Exp Brain Res 73:505-518.

Schnell L, Schwab ME (1990) Axonal regeneration in the rat spinal cord produced by an antibody against myelin-associated neurite growth inhibitors. Nature 343:269-272.

Schnell L, Schwab ME (1993) Sprouting and regeneration of lesioned corticospinal tract fibres in the adult rat spinal cord. Eur J Neurosci 5:1156-1171.

Schwab ME, Bartholdi D (1996) Degeneration and regeneration of axons in the lesioned spinal cord. Physiol Rev 76:319-370.

Tolbert DL, Der T (1987) Redirected growth of pyramidal tract axons following neonatal pyramidotomy in cats. J Comp Neurol 260:299-311.

Ugolini G, Kuypers HG (1986) Collaterals of corticospinal and pyramidal fibres to the pontine grey demonstrated by a new application of the fluorescent fibre labelling technique. Brain Res 365:211-227.

Wiesendanger R, Wiesendanger M (1982) The corticopontine system in the rat. II. The projection pattern. J Comp Neurol 208:227-238.

Z'Graggen WJ, Metz GA, Kartje GL, Thallmair M, Schwab ME (1998) Functional recovery and enhanced corticofugal plasticity after unilateral pyramidal tract lesion and blockade of myelin-associated neurite growth inhibitors in adult rats. J Neurosci 18:4744-4757. 